\title{
Long-Term Effects of Transcranial Magnetic Stimulation on Hippocampal Reactivity to Afferent Stimulation
}

\author{
Yechiel Levkovitz, ${ }^{1}$ Julia Marx, ${ }^{1}$ Nimrod Grisaru, ${ }^{2}$ and Menahem Segal ${ }^{1}$ \\ ${ }^{1}$ Department of Neurobiology, The Weizmann Institute, Rehovot 76100, Israel, and ${ }^{2}$ Mental Health Center, Ben Gurion \\ University, Beer Sheva 84105, Israel
}

Transcranial magnetic stimulation (TMS) has become a promising treatment of affective disorders in humans, yet the neuronal basis of its long-lasting effects in the brain is still unknown. We studied acute and lasting effects of TMS on reactivity of the rat hippocampus to stimulation of the perforant path. Application of TMS to the brain of the anesthetized rat caused a dose-dependent transient increase in population spike (PS) response of the dentate gyrus to perforant path stimulation. In addition, TMS caused a marked decrease in inhibition and an increase in paired-pulse potentiation of reactivity to stimulation of the perforant path. Also, TMS suppressed the ability of fenfluramine (FFA), a serotonin releaser, to potentiate PS response to perforant path stimulation. Chronic TMS did not affect single population spikes but caused an increase in paired-pulse potentiation, which was still evident 3 weeks after the last of seven daily TMS treatments. After chronic TMS, FFA was ineffective in enhancing reactivity to perforant path stimulation, probably because it lost the ability to release serotonin. In addition, the $\beta$ adrenergic receptor agonist isoproterenol, which caused an increase in PS in the control rats, failed to do so in the TMS-treated rats. These results indicate that TMS produces a long-term reduction in efficacy of central modulatory systems.

Key words: rat; hippocampus; serotonin; norepinephrine; TMS; perforant path
Transcranial magnetic stimulation (TMS) is a new noninvasive, safe, and painless method for the stimulation of the brain (Barker, 1991). Magnetic stimulation of the human brain is increasingly used for functional cortical mapping of primary motor pathway and speech areas and for the investigation of cortical function related to cognition in both health and disease states (Barker et al., 1986; Hallett and Cohen, 1989; Gates, 1995). It is also used as a contributing diagnostic tool in multiple sclerosis, motor neuron disease, facial spasm, stroke epilepsy, and peripheral nerve lesions (Jarratt, 1987). TMS has been suggested recently for the treatment of psychiatric disorders of mood and emotional dysfunction. Clinical studies in depressed patients (Hoflich et al., 1993; Grisaru et al., 1994; George et al., 1995) suggest antidepressant efficacy for TMS in humans. Despite its growing usage, few studies on possible long-term effects of TMS on central neurons have been reported (Wang and Scheich, 1996).

The hallmark of mood disorders is the emotional impairment associated with a serotonergic and noradrenergic dysfunction. Indeed, the great majority of the antidepressant drugs block the reuptake of serotonin or norepinephrine and downregulate postsynaptic $\beta$ adrenergic receptors. A less known impairment in depression involves cognitive and memory functions (Burt et al., 1995). The involvement of the hippocampus in cognitive and memory functions is well known, and its selective atrophy in depressed patients (Sheline et al., 1996) indicates that the impaired cognitive functions may be associated with the hippocampus. In addition, its dense innervation by serotonergic and nor-

\footnotetext{
Received Sept. 8, 1998; revised Dec. 2, 1998; accepted Feb. 1, 1999.

This work was supported in part by a grant from the Israel Center for Psychobiology, Jerusalem, Israel. We thank V. Greenberger for the histology, and Dr. R. H. Belmaker for the use of the magnetic stimulator.

Correspondence should be addressed to Menahem Segal, Department of Neurobiology, The Weizmann Institute, Rehovot 76100 Israel.

Copyright (C) 1999 Society for Neuroscience $\quad 0270-6474 / 99 / 193198-06 \$ 05.00 / 0$
}

adrenergic fibers arising from the midbrain raphe and the locus ceruleus, respectively, make the hippocampus a prime target for the study of TMS effects in brain tissue in relation to neuromodulation and depression.

The objectives of the present study are to characterize the short- and long-term effects of TMS on synaptic transmission and particularly on serotonergic and noradrenergic modulation of evoked synaptic activity in the hippocampus.

\section{MATERIALS AND METHODS}

Recording. Experiments were conducted with adult (3- to 4-months-old) male Wistar and Long-Evans rats of a local breeding colony. (The acute experiments were done with Long-Evans rats and the chronic experiments primarily with the Wistar rats. No differences in the effects of TMS between the two strains were found.) The rats were housed in a temperature-controlled room, three per cage, with $12 \mathrm{hr}$ light/dark cycle and access to food and water ad libitum. Rats were anesthetized with urethane ( $21 \%$ solution, $1.2 \mathrm{gm} / \mathrm{kg}$, i.p.) and placed in a stereotaxic apparatus. A bipolar, $125 \mu \mathrm{m}$ concentric stimulating electrode was placed in the perforant path (PP) (coordinates: $7.5 \mathrm{~mm}$ posterior to bregma, 3.0 $\mathrm{mm}$ lateral to the midline, depth of $3.5 \mathrm{~mm}$ ), and a single glass pipette (diameter of 2-3 $\mu \mathrm{m}$ ) containing $2 \mathrm{M} \mathrm{NaCl}$ was moved into the dentate gyrus of the dorsal hippocampus using an hydraulic microdrive as detailed elsewhere (Levkovitz and Segal, 1997). Electrode positions were optimized to record maximal population spikes (PS) in response to 100 $\mu$ sec pulse stimulation of the medial PP. Drugs were prepared in $2 \mathrm{M}$ $\mathrm{NaCl}$ at $10 \mathrm{~mm}$ concentrations from frozen stocks. When a drug pipette was used, it was introduced into the same location as the previous control pipette. This was confirmed by recording a depth profile (Fig. 1) while driving the pipette down to the reversal point of the EPSP in the granular cell layer. The location of the new pipette was verified by the production of the same EPSP to the same stimulation intensity. Drugs were allowed to diffuse into the recording area for several minutes before recording. Using this procedure, drug and control pipettes could be interchanged several times in the same experiment as described previously (Levkovitz and Segal, 1997). Evoked responses were amplified and filtered at $1 \mathrm{~Hz}-1$ $\mathrm{kHz}$ and stored for later analysis. In some experiments, a depth profile of the recording electrode was recorded as it was advanced in the dentate 

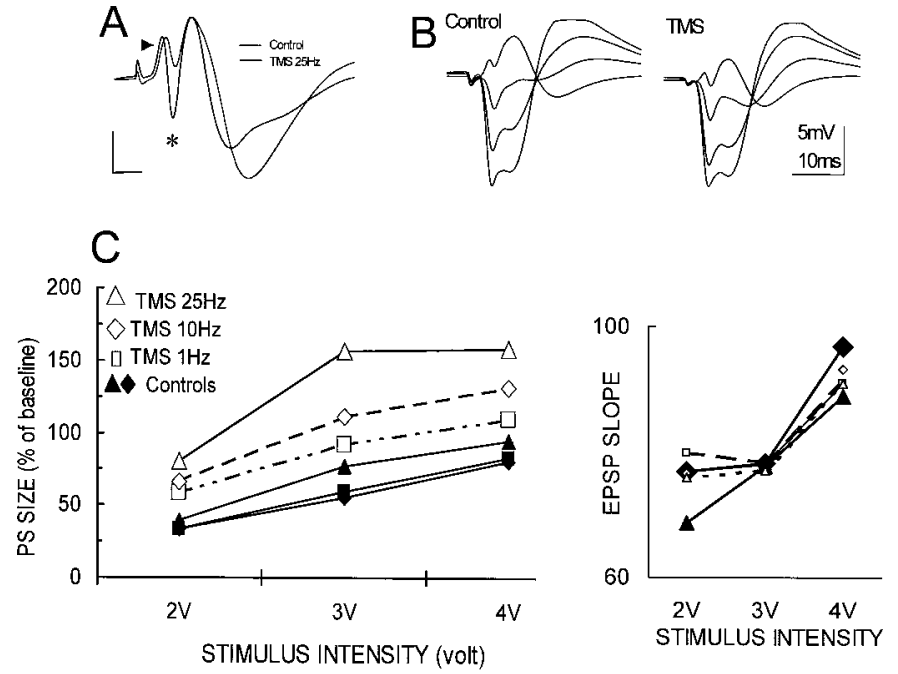

Figure 1. TMS potentiates population spike responses in the rat dentate gyrus. $A$, A sample record of population response to perforant path stimulation recorded before (thin line) and shortly after (thick line) a 25 $\mathrm{Hz}, 2 \mathrm{sec}$ train of magnetic stimulation. Note the similar slope of EPSP (arrowhead) and the larger population spike (asterisk) after TMS. Calibration: $5 \mathrm{msec}, 5 \mathrm{mV}$. $B$, Depth profile produced by stepping the recording pipette into the dentate granular layer. The traces, from bottom to top, are initially negative (down-going) in the molecular layer and reverse to positive EPSPs, with a negative-going population spike, at the granular layer (top traces). Note that after TMS the size of the EPSP is not changed, but the population spike is larger. $C$, Averages of responses taken before and after acutely applied TMS plotted as a function of stimulation intensity. Left, Magnitude of the population spike. Right, Slopes of EPSP. In both plots, the sizes of responses are expressed as percentage of control values. A TMS dose-dependent increase in population spikes is seen, with no significant differences in population EPSPs (right) to different stimulation intensities. The three control groups ( filled symbols) are for each treated group, taken before TMS treatment. SEs are smaller than the symbol sizes. All the treatment groups are different from each other significantly, but the control groups are similar to each other.

gyrus to verify that the recording electrode reached the same location before and after the application of TMS (Fig. 1). A twin pulse PP stimulus was delivered at three interpulse intervals $(15,30$, and $60 \mathrm{msec})$, and averages of 10 successive responses to a given intensity applied at a rate of $0.5 \mathrm{~Hz}$ were constructed. Paired-pulse response was quantified as the magnitude of the second over the first PS or the slope of the second EPSP over the first one.

TMS. One millisecond pulses were applied with a Cadwell (Kennewick, WA) Rapid Stimulator with a field intensity of 2.2 tesla [at $100 \%$ current intensity; flowing clockwise; calculated between 1-1.5 cm from the center of the coil (Cohen et al., 1990); estimated peak electric field strength of $660 \mathrm{~V} / \mathrm{m}$ ] through a $5 \mathrm{~cm}$ coil with a teardrop shape. Stimulation of 1,10 , and $25 \mathrm{~Hz}$ were applied for a total duration of $2 \mathrm{sec}$. Higher frequencies or duration caused overheating of the coil and were avoided. The coil was placed above the head, aligned with its center on the midline, equidistant between the bregma and lambda sutures along the longitudinal body axis. In all of the acute experiments, the recording electrode was removed just before and replaced back in the same location right after the magnetic stimulation. Recording of field EPSP and PS commenced as quickly as possible after the magnetic stimulation for variable duration up to $2 \mathrm{hr}$ after the stimulation. For the chronic experiments, awake rats were stimulated once daily for $7 \mathrm{~d}$ with a $2 \mathrm{sec}$ train of $25 \mathrm{~Hz}$, with the coil held against their skull above the dorsal hippocampus. Except for one rat, which was excluded from the analysis, none of the treated rats underwent epileptic seizures after the TMS treatment. Control rats were held the same way as the treated ones and were exposed to the same noise produced during the stimulation.

Analysis. Off-line measurements of the slopes of the EPSPs (in volts per second) and magnitudes of the maximal population spike (in millivolts) were made using averages of 10 successive responses to a given stimulation intensity applied at a rate of $0.1 \mathrm{~Hz}$. PS size and EPSP slope were measured as described previously (Richter-Levin and Segal, 1990). To standardize the calculations of the TMS and drug effects, the magnitudes of all responses were related to the responses to maximal baseline stimulation intensity used (100\%). Paired $t$ tests and ANOVA tests were used for statistical analysis when applicable.

\section{RESULTS}

\section{Acute effects}

Stimulation of the perforant path produced a typical intensitydependent population EPSP that is accompanied by negativegoing PS recorded in the granular layer of the anesthetized rat. At stimulation intensities that yielded $50 \%$ of the maximal response, the EPSP slope at baseline condition was $6.73 \pm 0.92 \mathrm{mV} / \mathrm{msec}$, and the PS was $2.31 \pm 0.57 \mathrm{mV}$. Application of TMS above the hippocampus did not have a significant effect on the slope of population EPSP in 22 rats tested with different stimulation intensities. In contrast, a dose-dependent increase in PS size was seen in these same rats (Fig. 1). The population spike increased significantly by $25 \pm 4.2,56 \pm 3.3$, and $81 \pm 4.9 \%$ above baseline values for TMS of 1,10 , and $25 \mathrm{~Hz}$, respectively, at PP stimulation that yielded $50 \%$ of the maximal response in control conditions (TMS of $1 \mathrm{~Hz}, p<0.001 ; n=5$; TMS of $10 \mathrm{~Hz}, p<0.0001 ; n=$ 5 ; TMS of $25 \mathrm{~Hz}, p<0.0001 ; n=7$ ) (Fig. $1 C$, for $3 \mathrm{~V}$ stimulation). The increase in population spike on a background of unchanged EPSP could be easily detected when plotting a depth profile along the path of the recording electrode in the dentate gyrus (Fig. 1B). This marked increase in spike size is therefore not likely to result from a change in afferent excitability or changes in postsynaptic glutamate receptor sensitivity but can be caused by a change in efficacy of feedforward inhibition in the dentate gyrus (Sloviter, 1991). Indeed, a similar change in spike size that is not accompanied by a change in EPSP slope could be seen after local application of the GABA-A receptor antagonist bicuculline (Levkovitz and Segal, 1997).

The possibility that TMS affects local GABAergic interneurons was tested more directly by examining the responses to a pairedpulse stimulation applied to the perforant path. In the normal case, the response to a second stimulus is totally suppressed if it follows a priming stimulus by $15 \mathrm{msec}$. A $30 \mathrm{msec}$ interpulse interval results in approximately the same response to the second as to the first stimulus, whereas longer interpulse intervals (60 and $90 \mathrm{msec}$ ) result in a twofold to threefold increase in response relative to the first one (Fig. 2). Three groups were tested with different TMS patterns. In the control pre-TMS condition, there was no difference between the groups. After TMS $(1,10$, and 25 $\mathrm{Hz}$ for $2 \mathrm{sec}$ each), there was a dose-dependent increase in reactivity to the second stimulus in a paired-pulse paradigm (Fig. 2), with the most striking effect being the total removal of inhibition at the short intertrial interval and its conversion to a marked potentiation: $55 \pm 7,90 \pm 7.5$ and $200 \pm 13 \%$ increase in PS2/PS1 ratio for TMS of 1,10 , and $25 \mathrm{~Hz}$, respectively $(n=5$, 5 , and 7 rats for the three groups, respectively) (Fig. 2B). This effect of TMS was the most consistent one and did not demonstrate any decay over time after the stimulation compared with the effect of TMS on the initial population spike magnitude, which tended to decay back to control level within 15-30 min after the magnetic stimulation (Fig. 3).

Serotonin has been shown to produce a marked enhancement of population spikes in the dentate granular layer without affecting population EPSP and is assumed to exert this effect by aselective reduction in the efficacy of GABAergic neurotransmis- 
Figure 2. Paired-pulse responses are drastically altered by TMS. $A$, Illustrations of responses to a twin pulse stimulation applied 15 and $30 \mathrm{msec}$ apart (arrows) in control (top) and after a $25 \mathrm{~Hz}, 2$ sec TMS (bottom). Note that the second population spike (asterisks) is eliminated in the control condition at $15 \mathrm{msec}$, and after TMS, it is actually larger than the response to the first stimulus. $B$, Summary of responses to paired-pulse stimulation, with the three intervals of 15,30 , and $60 \mathrm{msec}$, in control and after TMS applied at 1, 10, and $25 \mathrm{~Hz}$. A clear intensity-dependent, interpulse intervaldependent TMS effect on paired-pulse responses is seen. The controls are not different from each other. Each rat received a single TMS treatment and was measured before and after TMS.
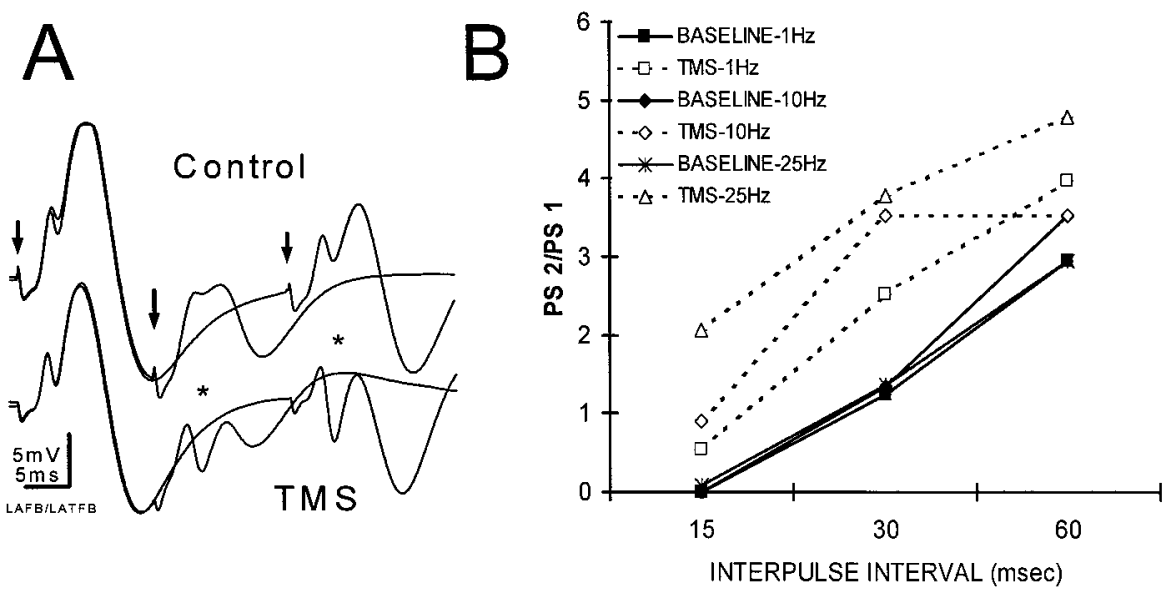

TIME COURSE $1 \mathrm{HZ}$

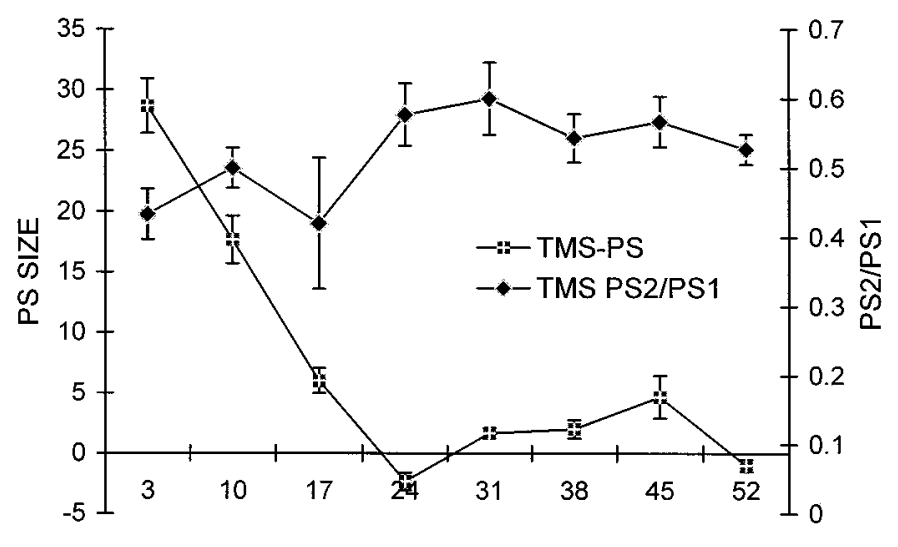

TIME

Figure 3. Time course of change in single population spikes and pairedpulse inhibition after TMS. The size of population spike, which increases on average by $30 \%$ after a short TMS, returns back to control value within 17 min after the TMS, whereas the blocked inhibition (ratio of PS2/PS1 at the $15 \mathrm{msec}$ interval) goes up from 0 in control condition (Fig. 2) to $\sim 0.5$ after TMS and remains elevated for the duration of testing $(52 \mathrm{~min}$ after acute TMS; $n=5$ ).

sion (Segal, 1990; Schmitz et al., 1995). One way to activate serotonergic receptors is by releasing serotonin from terminals using fenfluramine (FFA). Local or peripheral application of FFA have been shown to cause a 5-HT1a receptor-dependent 50-90\% increase in population spike without changing the EPSP slope (Richter-Levin and Segal, 1990). Applied either locally or peripherally after TMS $(10 \mathrm{~Hz}, 2 \mathrm{sec})$, FFA had no additional effect over that produced by TMS alone (Fig. 4). There was no effect of systemic $(n=3 ; 10-15 \mathrm{mg} / \mathrm{kg})$ or local FFA $(n=5)$ applied through the recording pipette on population spike after $10 \mathrm{~Hz}$ TMS. This may indicate that TMS and FFA share a common pathway for reducing GABA inhibition and enhancing population spikes. However, when applied after a higher intensity TMS (25 $\mathrm{Hz}, 2 \mathrm{sec}$ ), FFA (applied systemically in five rats and locally in five additional rats) actually reduced the magnitude of population spike significantly (to $45 \pm 4.8 \%$ of control) (Fig. $4 B$ ), indicating that TMS may have an effect on serotonergic release mechanisms or postsynaptic serotonin responses, in addition to its effect on GABAergic interneurons.
ACUTE FFA PS

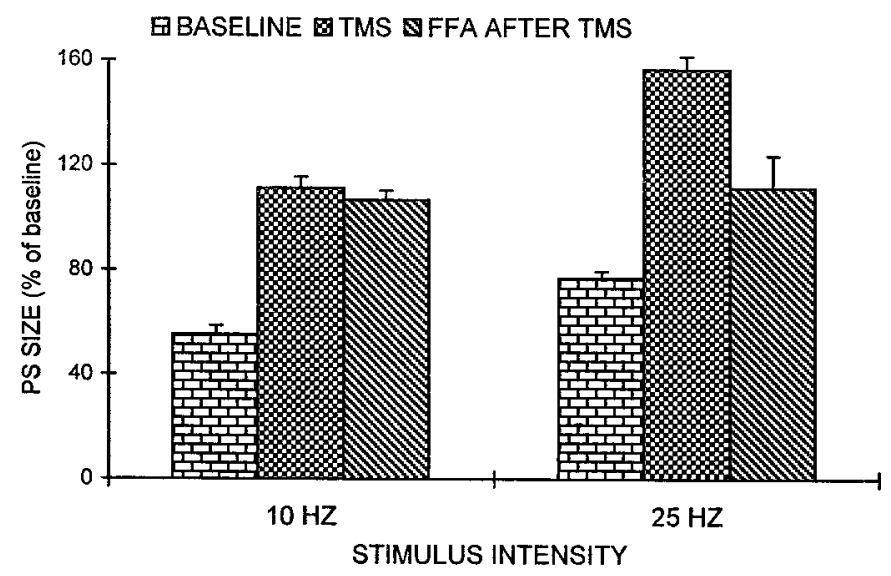

Figure 4. TMS blocks reactivity of the hippocampus to topical application of the serotonin releaser FFA. Evoked responses to perforant path stimulation are recorded with a control pipette before (baseline) and after TMS. An increase in population spike is recorded after both 10 (left) and 25 (right) Hz stimulation. The recording pipette is then replaced with one containing FFA. After $10 \mathrm{~Hz}$ TMS, the population spike response is increased nearly twofold, and no further increase in response to FFA is seen. After $25 \mathrm{~Hz}$ TMS, there is a large increase in population spikes, but FFA now causes a reduction in population spike relative to pre-FFA, post-TMS values.

\section{Chronic effects}

Rats were tested between 1 and 3 weeks after the last of a series of seven sessions of TMS. Control and treated rats were tested alternately. The results of three separate experiments were pooled in the final analysis because there were no differences between results of different experiments.

\section{Input-output relationships}

The input-output relationships were similar in 15 control and 18 TMS-treated rats. Both the population EPSPs and the population spikes maintained the same relationship to the stimulation intensity (Fig. 5). This indicates that the short-term effect, i.e., increase in PS/EPSP ratio (Fig. 1), seen in the acutely treated rats is not found in the chronically treated ones.

\section{Feedback inhibition}

The more sensitive assay for GABAergic inhibition is the pairedpulse response (Fig. 5B). As in the acute condition in which TMS 


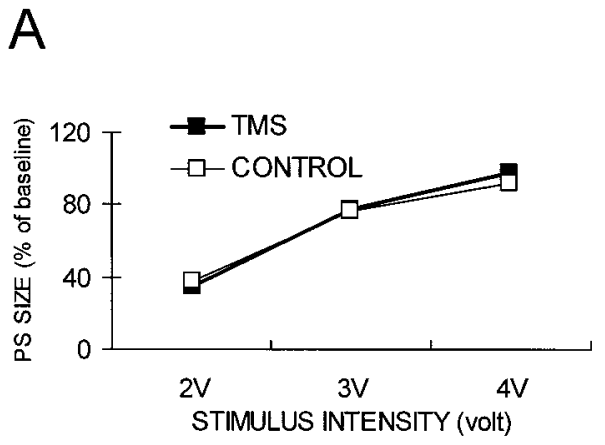

B

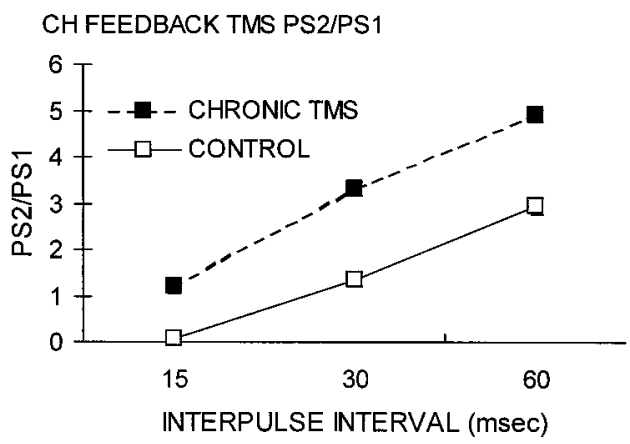

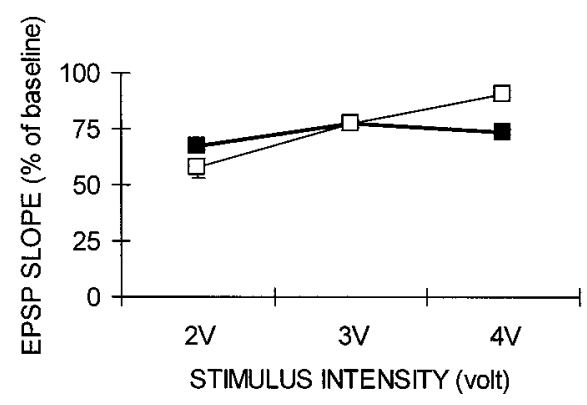

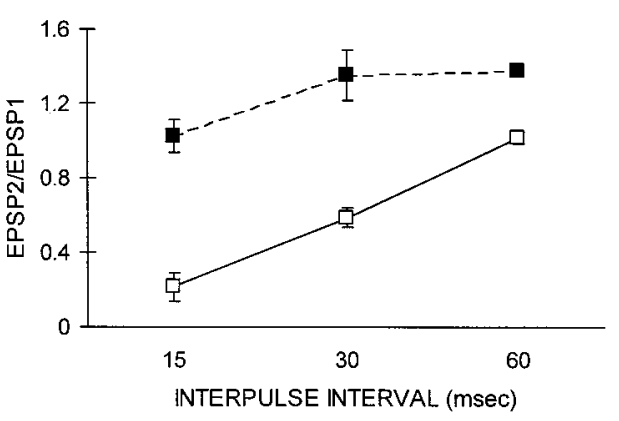

Figure 5. Chronic application of TMS does not affect single population spikes $(A)$ but affects paired-pulse responses $(B)$. Averages of recordings made 1-3 weeks after TMS application. $A$, There was no difference in the magnitudes of population spikes (left) between controls and TMS-treated rats. There were also no differences in the slopes of EPSPs in these same rats (right). The SEs are smaller than the symbols in these and the cases below. $B$, A marked increase in the magnitude of the response to the second of a pair of stimulation pulses applied at 15, 30, and 60 msec intervals. The differences compared with controls are significant at all intervals tested. Left, Population spikes. Right, Population EPSP slopes. Ordinate, The ratio of the second response to the first one. blocked paired-pulse inhibition for a longer time than the enhanced population spike to a single afferent stimulation, chronic TMS caused a long-lasting (for at least 3 weeks, the longest interval tested) marked reduction in paired-pulse inhibition (significant increase in PS2/PS1 for $3 \mathrm{~V}$ stimulation at all intervals tested in the $7 \mathrm{~d}$ post-TMS animals; $n=6$ ). This was especially pronounced at the short interpulse interval $(15 \mathrm{msec})$ in which the response to the second pulse in control rats was $8.5 \pm 4 \%$ of the response to the first stimulation, whereas in the chronic TMStreated rats, the response to the second stimulation was $120 \pm$ $8.6 \%$ of the response to the first stimulation (Fig. $5 B$ ). A significant potentiation of $200 \pm 29$ and $190 \pm 24 \%$ after chronic TMS stimulation compared with controls was found for interpulse interval of 30 and $60 \mathrm{msec}$, respectively. The potentiation was not restricted to the population spike, and a marked blockade of inhibition of the slope of the EPSP was seen, especially at the short interval (Fig. 5B). A similar reduction in inhibition of the slope of the EPSP was also seen in the acute case (data not shown). This indicates that the increase in population spikes at short intervals is likely to result from a reduction in dendritic inhibition in the dentate gyrus.

A similar reduction in paired-pulse inhibition was seen also 2 and 3 weeks after the chronic TMS treatment (14-d-treated, $n=$ 6 rats; 21-d-treated, $n=6$ rats; data not shown) .

\section{Serotonergic modulation of population spikes}

FFA did not affect population spikes in 18 chronic TMS-treated rats compared with the typical $70 \%$ increase in PS seen in matched control rats when applied either intraperitoneally $(n=6$ rats) or into the recording pipette ( $n=12$ rats). There was no increase in PS relative to control after local application of FFA in $7(n=6)$ and $21(n=6)$ d post-TMS treatment, and there was no effect of peripheral application of FFA in six rats tested $14 \mathrm{~d}$ after

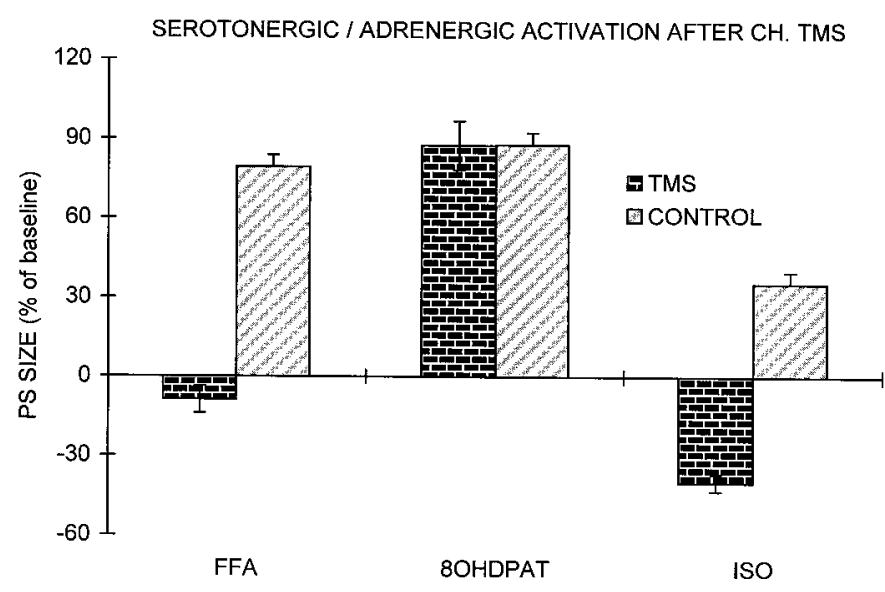

Figure 6. Differential effects of monoaminergic agents on population spikes evoked in response to perforant path stimulation after chronic TMS application. FFA applied intraperitoneally (left) produces a typical $80 \%$ increase in population spike response to the stimulation in control rats, although it does not affect responses in the TMS-treated rats. On the other hand 8-OH-DPAT, which was applied centrally at the site of recording, produced the same elevation of population spike in both control and TMS-treated rats. Isoproterenol, a $\beta$ agonist applied locally in the recording pipette, produced a modest but significant $30 \%$ increase in population spike in control rats (right) but produced a significant reduction in population spike size in the TMS-treated rats. See Results for further details.

TMS. In fact, in some cases, FFA actually caused a reduction in population spike size up to $40 \pm 12 \%$ when applied locally (Fig. 6). The lack of effect of FFA can be caused either by a desensitized postsynaptic serotonergic receptor or by a reduced release of serotonin from its presynaptic terminals. In the latter case, direct application of a serotonin agonist through the recording 
pipette should mimic the effect of FFA found in the normal case as seen previously (Levkovitz and Segal, 1997). Indeed, topical application of 8-hydroxy-2-dipropylaminotetralin (8-OH-DPAT), a 5-HT1a serotonin receptor agonist (Hall et al., 1985), caused a marked increase of $81 \pm 3 \%$ in population spike size, an effect that was indistinguishable from the effect of 8-OH-DPAT found in control rats (Fig. 6). The increase in PS after local application of 8-OH-DPAT was significant ( $3 \mathrm{~V}$ stimulation intensity, $p<$ $0.03 ; n=6)$.

\section{Noradrenergic modulation}

Local application of isoproterenol in the hippocampal granular layer of control rats showed an increase in population spike $(35.2 \pm 3.2 \%$ for $3 \mathrm{~V} ; p<0.05 ; n=6)$ (Fig. 6 , right) that was not accompanied by a change in population EPSP (data not shown). Local application of isoproterenol in chronic TMS-treated rats caused significant reduction in PS $(-20.5 \pm 4.5 \%$ for $3 \mathrm{~V} ; p<$ $0.05 ; n=6$ ) relative to baseline response with no significant change in the EPSP (data not shown).

\section{DISCUSSION}

The present results demonstrate long-lasting effects of TMS on reactivity of the hippocampus to stimulation of its main excitatory afferent pathway arriving from the entorhinal cortex, the perforant path. Three effects of TMS were found. First, there was a short-lasting increase in population spike response to perforant path stimulation without affecting the population EPSP in this pathway. This effect subsided within minutes after the TMS stimulation and was not seen in chronically treated rats. The more persistent effects, which were seen in both the acutely treated and the chronically treated rats, consisted of a marked reduction in paired-pulse inhibition seen normally with short $(15 \mathrm{msec})$ interpulse intervals and an increase in paired-pulse potentiation seen with longer interpulse intervals. In addition, TMS caused a large and prolonged suppression of the reactivity of the hippocampus to the serotonin-releasing drug FFA, which enhances population spike response to afferent stimulation in the normal rat brain. Also, the direct effect of the $\beta$ adrenergic agonist isoproterenol was significantly reduced. Interestingly, these two chronic effects of TMS serve opposite functions in the hippocampus; the first one, reduced feedback-inhibition and increased paired-pulse potentiation, causes an increase in excitability, whereas the second one, reducing efficacy of FFA and isoproterenol, acts to decrease excitability of the hippocampus. In these respects, our results are similar to those of previous studies in which both excitatory and inhibitory effects of TMS can be found (Wang and Scheich, 1996).

The cellular and molecular basis of these actions of TMS are not entirely clear. An increase in population spike size that is not accompanied by a change in EPSP is assumed to result from a reduction in local feedforward modulation by GABAergic neurons of the spike-generating mechanism. Such an effect is caused by the serotonin releaser FFA, which suppresses feedforward interneuron activity and is assumed to increase reactivity of the hippocampus to afferent stimulation (Richter-Levin and Segal, 1990, 1991). The initial acute effect of TMS to increase population spikes may be mediated by release of serotonin, because it has been found that TMS causes an acute reduction in 5-HT content in the hippocampus, probably by releasing it from terminals (Ben-Shachar et al., 1997). If so, this may explain why FFA is no longer effective and why the serotonin 5-HT1a agonist 8-OHDPAT is able to potentiate the response to the stimulation. It indicates that the suppressed FFA action is attributable to the lack of release of serotonin from terminals and not to altered postsynaptic response to the released serotonin. It seems that TMS may have a long-term action on release of serotonin, but the molecular mechanisms underlying this effect are not clear as yet.

In comparison, the $\beta$ adrenergic agonist isoproterenol, which acts directly on the postsynaptic receptor, loses its action in the TMS-treated brains. This effect is consistent with a recent report on the downregulation of $\beta$ adenoreceptors after TMS (Fleischmann et al., 1996) and may hint to a long-term effect of TMS on biochemical processes in the brain.

The lasting effect of TMS on paired-pulse response indicates that TMS may have a primary action on local circuit neurons activated in the paired-pulse protocol. The marked effect of TMS on the population EPSP in the paired-pulse protocol, which points to a dendritic location of TMS action, is distinct from the effect on serotonin terminals assumed to act on feedforward somatic inhibitory connection (Buzsaki, 1984; Freund and Buzsaki, 1996). The lack of obvious morphological effects of TMS or the lack of signs of chronic epilepsy also mitigate against a chronic reduction in inhibitory connections in the hippocampus, which may result in epilepsy and cell loss. On the other hand, the simultaneous reduction in the ability to increase excitability by a monoaminergic action indicates that the overall action of TMS may be to decrease rather than increase excitability of the hippocampus, at least with respect to feedforward modulation of population discharges.

Regardless of the molecular mechanisms, the reduction in noradrenergic and serotonergic functions in the hippocampus of chronically TMS-treated rats may have important implications for understanding the biological basis of clinical TMS and depression. There are similar effects on monoamines with other treatments of depression (e.g., with electroconvulsive shock treatment). The ability to control and quantify the physiological effects of TMS exceeds that seen with the other treatments; hence, its use will contribute more to the understanding the roles of serotonin and noradrenaline in regulation of mood disorders and memory functions.

In the chronically treated rats, there were significant changes in motility in an open field, a sensitive indicator of fear in the tested rat, as well as an increase in pain sensitivity, another indicator of emotionality and hyper-reactivity (our unpublished observations). Neither of these effects is intuitively associated with beneficial action of TMS reported in human patients (Grisaru et al., 1994), yet both of these functions are regulated by monoamine systems affected by the TMS treatment. Also, these behavioral effects are not necessarily related to our observed changes in hippocampal-evoked potentials because the TMS may have affected other neuronal systems in the brain, as well.

The relevance of the current studies to the understanding of TMS effects in depression is not immediately obvious. The size of the rat brain and the fact that TMS may very well stimulate the entire brain is certainly different from TMS as applied to the human brain in which the stimulation affects a restricted region of the brain (Weissman et al., 1992). Intuitively, the hippocampus may not be the primary locus associated with depression or the preferred site for application of TMS in depressed patients. However, the fact that the hippocampus shrinks in depressed patients (Sheline et al., 1996) and that there are cognitive dysfunctions in depressed patients (Burt et al., 1995) indicate that the hippocampus may be related to some aspects of depression. The wealth of information on the electrophysiology of the hippocampus and the neuromodulatory actions of monoamines there 
make it a convenient, but also justified, brain region for the study of possible effects of TMS on neuromodulation and depression.

\section{REFERENCES}

Barker AT (1991) An introduction to the basic principles of magnetic nerve stimulation. J Clin Neurophysiol 8:26-37.

Barker AT, Freeston IL, Jalinous R, Jarratt JA (1986) Clinical evaluation of conduction time measurements in central motor pathway using magnetic stimulation of human brain. Lancet 1:1325-1326.

Ben-Shachar D, Belmaker RH, Grisaru N, Klein E (1997) Transcranial magnetic stimulation induces alterations in brain monoamines. J Neural Transm 104:191-197.

Burt DB, Zembar MJ, Niederehe G (1995) Depression and memory impairment: a meta analysis of the association, its pattern and specificity. Psychol Bull 117:285-305.

Buzsaki G (1984) Feed-forward inhibition in the hippocampal formation. Prog Neurobiol 22:131-153.

Cohen LG, Roth BJ, Nilsson J, Dang N, Panizza M, Bandinelli S, Friauf W, Hallett M (1990) Effects of coil design on delivery of focal magnetic stimulation. Technical considerations. Electroencephalogr Clin Neurophysiol 75:350-357.

Fleischmann A, Sternheim A, Etgen AM, Li C, Grisaru N, Belmaker RH (1996) Transcranial magnetic stimulation downregulates $\beta$-adrenoreceptors in rat cortex. J Neural Transm 103:1361-1366.

Freund TF, Buzsaki G (1996) Interneurons of the hippocampus. Hippocampus $6: 347-370$.

Gates JR (1995) Transcranial magnetic stimulation. Neuroimaging Clin N Am 5:711-720.

George MS, Wassermann EM, Williams WA, Callahan A, Ketter TA, Basser P, Hallett M, Post RM (1995) Daily repetitive transcranial magnetic stimulation (rTMS) improves in refractory depression. NeuroReport 6:1853-1856.

Grisaru N, Yaroslavsky U, Abarbanel J, Lamberg T, Belmaker RH (1994) Transcranial magnetic stimulation in depression and schizophrenia. Eur Neuropsychopharmacol 4:287-288.

Hall MD, Mestikaway S, Emerit MB, Pichat L, Hammon M, Gozlan H
(1985) 8-OH-DPAT binding to pre- and postsynaptic 5-HT sites in various region of the brain. J Neurochem 44:1685-1696.

Hallett M, Cohen LG (1989) Magnetism: a new method for stimulation of nerve and brain. JAMA 262:538-541.

Hoflich G, Kasper S, Hufnagel A, Ruhrmann S, Moller HJ (1993) Application of transcranial magnetic stimulation in treatment of drugresistant major depression - a report of two cases. Hum Psychopharmacol 8:361-365.

Jarratt JA (1987) Magnetic stimulation: a new method of investigating the nervous system. Neurosurgery 20:100-109.

Levkovitz Y, Segal M (1997) Serotonin 5-HT1A receptors modulate hippocampal reactivity to afferent stimulation. J Neurosci 17: 5591-5598.

Richter-Levin G, Segal M (1990) Effects of serotonin releasers on the dentate granular cell exitability in the rat. Exp Brain Res 82: 199-207.

Richter-Levin G, Segal M (1991) The effects of serotonin depletion and raphe grafts on hippocampal electrophysiology and behavior. J Neurosci 11:1585-1596.

Schmitz D, Empson RM, Heinemann U (1995) Serotonin reduces inhibition via 5-HT1a receptors in area CA1 of the rat hippocampal slices in vitro. J Neurosci 15:7217-7225.

Segal M (1990) Serotonin attenuates a slow inhibitory postsynaptic potential in rat hippocampal neurons. Neuroscience 36:631-641.

Sheline YI, Wang PW, Gado MH, Csernansky JG, Vannier MW (1996) Hippocampal atrophy in recurrent major depression. Proc Natl Acad Sci USA 93:3908-3913.

Sloviter RS (1991) Feedforward and feedback inhibition of hippocampal principal cell activity evoked by perforant path stimulation: GABAmediated mechanisms that regulate excitabitlity in vivo. Hippocampus 1:31-40.

Wang H, Scheich H (1996) LTD and LTP induced by transcranial magnetic stimulation in auditory cortex. NeuroReport 7:521-525.

Weissman JD, Epstein CM, Davey KR (1992) Magnetic brain stimulation and brain size: relevance to animal studies. Electroencephalogr Clin Neurophysiol 85:215-219. 